\title{
Voucher für Glasfaseranschlüsse: Warum Bund und Länder auf sie verzichten sollten
}

\author{
Seit einigen Jahren wird von verschiedenen Seiten empfohlen, nachfrageseitig den Ausbau \\ von Glasfaseranschlüssen in Deutschland mit „Gigabit-Vouchern“ staatlich zu subventionieren. \\ Solche Gutscheine weisen jedoch erhebliche Nachteile auf. Bundes- und Landesregierungen \\ sollten sie deshalb nicht einsetzen.
}

Im Koalitionsvertrag vom März 2018 geben CDU, CSU und SPD ein ambitioniertes Ziel für die Versorgung Deutschlands mit leistungsstarken Breitbandanschlüssen vor: Bis 2025 will man „den flächendeckenden Ausbau mit Gigabit-Netzen ... mit Glasfaser in jeder Region und in jeder Gemeinde, möglichst direkt bis zum Haus"1 erreichen. Tatsächlich waren Ende 2019 erst 4,39 Mio. $(10,6 \%)$ private Haushalte in Deutschland an leistungsstarke Netze angeschlossen, die Glasfaserkabel bis zum Gebäudekeller (Fiber-To-The-Building; FTTB) oder bis zur Geräteanschlussdose in der Wohnung (Fiber-To-TheHome; FTTH) einsetzen. Von den technisch anschließbaren Haushalten hatten nur 1,49 Mio. (33,8\%) auch einen FTTB/H-Anschlussvertrag mit einem Netzbetreiber unterzeichnet (vgl. Abbildung 1). Angesichts des seit Jahren niedrigen Verhältnisses zwischen der Zahl der tatsächlichen Kunden und der Zahl der technisch möglichen FTTB/H-Anschlüsse überrascht es nicht, dass man nach Wegen zur Erhöhung der „Take-Up-Rate“ bei Gigabit-Anschlüssen sucht.

Vor diesem Hintergrund empfiehlt die Monopolkommission in ihrem im Dezember 2019 veröffentlichten 11. Sektorgutachten Telekommunikation ${ }^{2}$ - wie schon zwei Jahre zuvor $^{3}$ - auf Bundesebene als Ergänzung zur Subventionierung des angebotsseitigen Ausbaus von Anschluss-

(C) Der/die Autor(en) 2020. Open Access: Dieser Artikel wird unter der Creative Commons Namensnennung 4.0 International Lizenz (https:// creativecommons.org/licenses/by/4.0/deed.de) veröffentlicht.

Open Access wird durch die ZBW - Leibniz-Informationszentrum Wirtschaft gefördert.

1 CDU, CSU, SPD: Koalitionsvertrag vom 12.3.2018, 19. Legislaturperiode, S. 38, https://www.cdu.de/system/tdf/media/dokumente/ koalitionsvertrag_2018.pdf?file=1 (19.12.2019). Zur Einordnung der netzpolitischen Ziele in diesem Vertrag siehe J. Sickmann, A. Neumann: „Digitalland“ Deutschland?, in: Wirtschaftsdienst, 98. Jg. (2018), H. 5, S. 333-335.

2 Monopolkommission: Staatliches Augenmaß beim Netzausbau, Bonn 2019, S. 61-64.

3 Dies.: Telekommunikation 2017: Auf Wettbewerb bauen!, Bonn 2017, S. 80-81. netzen der nächsten Generation in „weißen Flecken“4 eine nachfrageseitige Förderung von FTTB/H-Anschlüssen bei privaten Haushalten sowie kleinen und mittleren Unternehmen (KMU) durch „Gutscheine sowohl für den Abschluss eines Vertrags als auch die Errichtung eines Glasfaserhausanschlusses"5. Die Monopolkommission steht mit ihrem Plädoyer zugunsten von derartigen, auch als Gigabit-Vouchern bezeichneten, Gutscheinen nicht allein. Seit Anfang 2016 betonen Bundes- und Landesministerien, Wirtschaftsverbände wie Bundesverband der Deutschen Industrie (BDI), Bundesverband Breitbandkommunikation (BREKO) oder Verband der Anbieter von Telekommunikations- und Mehrwertdiensten (VATM), Berater und Wissenschaftler ${ }^{6}$ sowie jüngst auch die Fraktion der FDP im Bundestag in einem plakativ mit „Smart Germany - Gigabit-Gutscheine für den Breitbandausbau“ über-

4 Seit Oktober 2015 können privat- und kommunalwirtschaftliche Netzerbauer/-betreiber vom Bund Zuschüsse für die Erschließung von Gebieten erhalten, die zuvor nicht an leistungsstarke Festnetze angebunden waren (,weiße Flecken“); vgl. Monopolkommission: Staatliches Augenmaß ..., a.a. O., S. 57 (Fußnote 161), S. 50-56; T. J. Gerpott: Breitbandsubventionen des Bundes 2015-2017, in: ifo Schnelldienst, 70. Jg. (2017), H. 20, S. 16-22. Ähnliche Programme haben auch die Bundesländer eingerichtet.

5 Monopolkommission: Staatliches Augenmaß ..., a.a. O., S. 6.

6 Vgl. beispielhaft Bundesministerium für Wirtschaft und Energie: Weissbuch digitale Plattformen, Berlin 2017, S. 85 und 109; W. Briglauer, P. Schmitz: Gutachten zur ökonomischen und rechtlichen Sinnhaftigkeit von nachfrageseitigen Förderungen im Ausbau moderner Breitbandnetze über „Voucher-Systeme“, Mannheim 16.9.2019; Bundesverband der Deutschen Industrie: Gigabit-Netze für Deutschland, Berlin 10.10.2018; BREKO, VATM: Gigabit-Voucher für Bürger und Unternehmen, Berlin 26.9.2019; BREKO, VATM: Gigabit-Voucher für effizienten Ausbau, Bonn, 9.12.2019; BUGLAS: Grundsatzpapier zur Zukunft der Breitbandförderung, Bonn 13.7.2018; S. Haupt, S. Jung, J. Lichter, F. C. May: Infrastruktur der Zukunft, Düsseldorf 2019, S. 183-185; J. Kühling, F. Toros: Rechtliche Herausforderungen bei der Schaffung von Anreizen für einen flächendeckenden Ausbau von Glasfaserinfrastrukturen, Regensburg 25.7.2019, S. 90-104; Landesregierung Sachsen-Anhalt: Digitale Infrastrukturen der Zukunft, Magdeburg 14.5.2019, S. 10; VATM: Wie Voucher beim Umstieg auf FTTB/H helfen, Berlin 15.10.2018; C. Wernick, S. Strube Martins, C. M. Bender, C.-I. Gries: Markt- und Nutzungsanalyse von hochbitratigen TK-Diensten für Unternehmen der gewerblichen Wirtschaft in Deutschland, Bad Honnef 16.3.2016, S. 82. 
Abbildung 1

Bestand Fiber-To-The-Building/-Home-Anschlüsse in Deutschland

in Tausend, jeweils zum Jahresende, Zahl der Haushalte jeweils $100 \%$

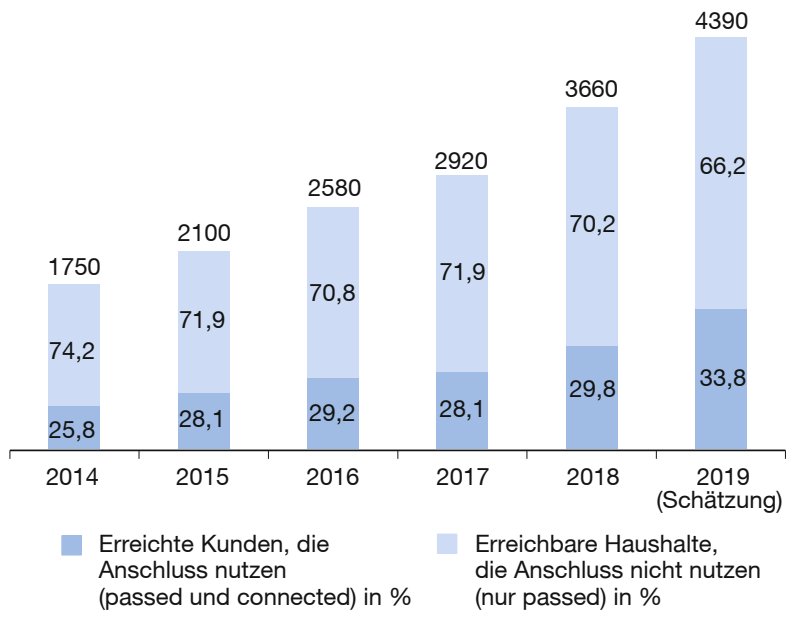

Quellen: Dialog Consult; Prof. Gerpott Analysen.

schriebenen Beschlussantrag ${ }^{7}$ eher undifferenziert Vorteile einer Subventionierung von FTTB/H-Anschlüssen durch Ausgabe von Gutscheinen. Mehrere juristische Analysen kommen zu dem Schluss, dass Glasfaser-Voucher mit europäischen und deutschen subventions- und telekommunikationsrechtlichen Vorschriften vereinbar sind. ${ }^{8}$ Die uneingeschränkt positive Sicht spiegelt sich auch darin wider, dass die nordrhein-westfälische Landesregierung Ende 2018 den Betrag von 1 Mio. Euro für ein auf Glasfaseranschlüsse ausgerichtetes Gutschein-Programm in ihr Landeshaushaltsgesetz 2019 eingestellt hat. ${ }^{9}$

Demnach scheinen Gigabit-Voucher ein exzellentes Mittel zu sein, um den in internationalen Vergleichen konstatierten Rückstand insbesondere ländlicher Regionen Deutschlands beim Ausbau und der Nachfrage von FTTB/H-Anschlüssen zu verringern. Eine genauere Betrachtung lässt aber ernsthafte Bedenken aufkommen, ob der Augenschein nicht trügt. Mit den Gutscheinen sind nämlich gravierende Probleme verbunden, denen in der öffentlichen Diskussion bislang wenig Beachtung geschenkt wird. Ziel dieses Bei-

7 Vgl. Deutscher Bundestag: Drucksache 19/14048 vom 16.10.2019; http://dipbt.bundestag.de/dip21/btd/19/140/1914048.pdf (19.12.2019).

8 Vgl. W. Briglauer, P. Schmitz, a.a. O., S. 34; A. Neumann: Optionen für die Ausgestaltung eines rechtlich abgesicherten Anspruchs auf schnelles Internet aus telekommunikationsrechtlicher Sicht, Bonn 1.2.2019, S. 23; S. Rennekamp: Machbarkeitsstudie zur Förderung der Nachfrage zugunsten zukunftssicherer, ultraschneller Glasfaseranschlüsse bis ins Gebäude, Hamburg 17.7.2018, S. 10-11.

9 Vgl. Beschlussempfehlung und Bericht des Haushalts- und Finanzausschusses des Landtages Nordrhein-Westfalen (Drucksache 17/4450 vom 6.12.2018), S. 75, https://www.landtag.nrw.de/portal/ WWW/dokumentenarchiv/Dokument/MMD17-4450.pdf (19.12.2019).
Univ.- Prof. Dr. Torsten J. Gerpott leitet den Lehrstuhl für Unternehmens- und Technologieplanung an der Mercator School of Management Duisburg der Universität Duisburg-Essen.

trags ist es, nach einer kurzen Beschreibung dessen, was unter Voucher-Konzepten für Glasfaseranschlüsse verstanden wird, diese Nachteile zu verdeutlichen.

\section{Gigabit-Gutscheine: Gegenstandspräzisierung}

Bei "Gigabit-Vouchern“ geht es um niedrige, von der öffentlichen Hand finanzierte, einmalige, nicht zurück zu zahlende Förderbeträge von zumeist nicht mehr als 500 Euro pro Empfänger, mit denen ein (eher kleiner) Teil der Kosten erstattet wird, die auf Nachfragerseite durch einen FTTB/H-Anschluss entstehen. Als Zielgruppen für den Gutscheinempfang gelten Hauseigentümer, private Endkunden und KMU. Sie reichen die Zuschussberechtigung in Form eines Gutscheins bzw. Subventionsantrags bei einer staatlichen Organisation ein - in Betracht kommt dafür das Bundesamt für Wirtschaft und Ausfuhrkontrolle (BAFA) oder die Kreditanstalt für Wiederaufbau (KfW). Bedingung für die Auszahlung des Vouchers ist, dass sie von einem Netzbetreiber ihrer Wahl mit einem FTTB/HAnschluss versorgt wurden. Dabei werden zwei Typen von „Versorgung" bzw. Gutscheinen unterschieden. ${ }^{10}$ Bei der als „Anschluss-Voucher" bezeichneten Variante erhalten Gebäudeeigentümer die Förderung nach der Verlegung von Glaserfaser auf der Stichstrecke von der Grundstücksgrenze bis zum Haus durch einen FTTB/H-Netzbetreiber. Bei dem Modell "Vertrags-Voucher" empfangen Endkunden den Zuschuss nach Unterzeichnung eines Vertrags mit einer längeren Laufzeit (vorgeschlagen werden häufig mindestens zwei Jahre) über die Bereitstellung eines glasfaserbasierten Netzanschlusses mit besonders hoher Bandbreite (empfohlen werden derzeit zumeist Empfangsgeschwindigkeiten von mehr als $250 \mathrm{Mbit} / \mathrm{s}$ ).

Voucher-Konzepte sehen durchweg vor, Gigabit-Gutscheine auf Gebiete zu beschränken, „in denen noch keine gigabitfähigen Infrastrukturen vorhanden sind"11. So

10 Zusätzlich werden zur staatlichen Subventionierung der gebäudeinternen Nachrüstung mit für sehr hohe Geschwindigkeiten geeigneten Kabeln vom Übergabepunkt des gebäudeexternen Netzes (typischerweise im Keller) bis zu Anschlussdosen in den Räumen der Wohnungen größerer Immobilien „Inhouse-Voucher" diskutiert. Auf eine getrennte Betrachtung des dritten Gutscheintyps wird hier aus Platzgründen verzichtet. Grundsätzlich gelten die Einwände gegen Gigabit-Voucher-Programme ebenfalls für diesen Typ.

11 Monopolkommission: Staatliches Augenmaß ..., a. a. O., S. 63-64. 
soll darauf hingewirkt werden, die Wirtschaftlichkeit des Ausbaus von FTTB/H-Netzen gezielt in Regionen zu verbessern, die sonst nicht erschlossen worden wären. Investitionszuträgliche Rentabilitätssteigerungen sollen sich ergeben, weil Netzbetreiber durch Voucher Deckungsbeiträge mit zusätzlichen Kunden erzielen könnten, die ohne die mit Vouchern einhergehende Senkung der von innen zu zahlenden Preise keinen Gigabit-Vertrag unterzeichnet hätten. In diesem Zusammenhang ist allerdings darauf hinzuweisen, dass Netzbetreiber zusammenhängende größere, dicht besiedelte Neubaugebiete heute im Regelfall mit FTTB/H als Stand der Technik ausstatten. Folglich ist eine Ausrichtung von Vouchern auf Gebiete, in denen Bestandsimmobilien nicht an FTTB/H-Netze oder mit Glasfaser aufgerüstete Kabelnetze angebunden sind, aus Sicht des Subventionsgebers zweckmäßiger. ${ }^{12}$

Die auf den Bund zukommenden Kosten von VoucherProgrammen zur Unterstützung der FTTB/H-Anbindung von Privathaushalten in Regionen, in denen keine GigabitInfrastrukturen verfügbar sind, werden für Deutschland auf 1,5 Mrd. bis 3,0 Mrd. Euro geschätzt, wenn man den Zuschuss auf 500 Euro pro Empfänger begrenzt. ${ }^{13}$

\section{Probleme von Gigabit-Gutscheinen}

\section{Geringe und ineffiziente Nachfrageausweitung}

Gigabit-Voucher sollen dazu beitragen, die Nachfrage nach FTTB/H-Anschlüssen erheblich zu steigern. Empirische Studien belegen jedoch, dass der Absatz von Breitbandanschlüssen an Privathaushalte auf Preisveränderungen nur unterproportional reagiert. ${ }^{14}$ Die Verkaufschancen von Gigabit-Anschlüssen werden viel stärker von ihrer Notwendigkeit für ein reibungsloses Funktionieren privat oder gewerblich wichtiger Anwendungen beeinflusst. ${ }^{15}$ Da Gutschein-Konzepte außerdem einhellig vorsehen, mit dem Förderungsbetrag nur einen kleinen Teil der Gesamtkosten eines FTTB/H-Anschlusses für

12 Vgl. zum Einbezug von Neubaugebieten in Voucher-Programme ANGA Der Breitbandverband: Stellungnahme zur Konsultation des Bundesministeriums für Verkehr und digitale Infrastruktur, Köln 7.9.2018, S. 5; W. Briglauer, P. Schmitz, a. a. O., S. 6-7, 13 und 30.

13 Vgl. VATM, a.a. O., S. 2-3; VATM: Warum Voucher?, Berlin 10.7.2018, S. 3. 14 Vgl. zur Zusammenfassung einschlägiger Befunde P. Koutroumpis: The economic impact of broadband, London 27.4.2018, S. 12; M. Svigelj, N. Hrovatin, J. Zoric: Are Internet users in Slovenia willing to pay for fast broadband and what drives them to migrate?, in: Economic Research - Ekonomska Istraživanja, 32. Jg. (2019), H. 1, S. 1003.

15 Vgl. z.B. L. Abradi, C. Cambini: Ultra-fast broadband investment and adoption, in: Telecommunications Policy, 43. Jg. (2019), H. 3, S. 191; T. J. Gerpott: Relative fixed Internet connection speed experiences as antecedents of customer satisfaction and loyalty, in: Management \& Marketing, 13. Jg. (2018), H. 4, S. 1153; M. Svigelj, N. Hrovatin, J. Zoric, a.a.O., S. 1003; M. Svigelj, N. Hrovatin: Analysis of household broadband technology preferences in Slovenia, in: Eurasian Business Review, 9. Jg. (2019), H. 4, S. 417.
Gebäudeeigentümer oder Endkunden abzudecken, ist zu erwarten, dass Voucher lediglich eine geringe Nachfrageausweitung bewirken werden. Wenn die Nachfrageeffekte bei niedrigen Gutscheinbeträgen schwach und damit Verbesserungen der Wirtschaftlichkeit des FTTB/H-Ausbaus marginal sind, dann motivieren sie Netzbetreiber kaum dazu, in großem Maßstab zusätzliche Gebiete mit Glasfaser zu erschließen.

Um eine merkliche Nachfrageausweitung zu bewirken, müsste der Subventionsbetrag je Gutschein relativ zu den Gesamtkosten eines FTTB/H-Anschlusses auf Kundenseite hoch sein. Eine solche Voucher-Gestaltung ist in der Praxis nicht nur aufgrund fiskalischer Engpässe unwahrscheinlich. Sie ist auch insoweit ineffizient, als mindestens der gleiche Anstieg mit geringerem Finanzmitteleinsatz durch Förderung von sehr "bandbreitenhungrigen“ Diensten, die aus Nutzersicht attraktiv sind, erreicht werden kann. ${ }^{16}$

\section{Problematische Verteilungseffekte}

Gigabit-Voucher haben fragwürdige verteilungspolitische Konsequenzen. Sie resultieren erstens daraus, dass das verfügbare Einkommen von Privathaushalten positiv mit der nachgefragten Bandbreite von Breitbandanschlüssen, der Wahrscheinlichkeit der Adoption eines Glasfaseranschlusses sowie der Zahlungsbereitschaft für einen Glasfaseranschluss korreliert. ${ }^{17}$ Der Anteil der Berechtigten, der einen Gigabit-Gutschein einlöst, wird deshalb in der Gruppe der Haushalte mit höherem Einkommen den Anteil in der Gruppe mit niedrigem Einkommen übersteigen. Zweitens begünstigen Voucher besonders private Haushalte auf dem Land, weil es in dünn besiedelten Regionen bislang an FTTB/H-Angeboten mangelt. In ländlichen Gebieten haben Ein- und Zweifamilienhäuser, die von Bürgern der Mittel- oder Oberschicht bewohnt werden, eine relativ größere Bedeutung als in Städten. Insgesamt werden folglich durch Voucher vergleichsweise wohlhabende Haushalte von der Gesamtheit aller Steuerzahler, die zu einem erheblichen Teil materiell schlechter gestellt sind als die Subventionsempfänger, unterstützt.

16 Vgl. die in Fußnote 15 angeführten Quellen.

17 Vgl. L. Abradi, C. Cambini, a.a. O., S. 187 und 192-193; K. BensassiNour, J. Liang: Discrete choice analysis of demand for broadband in France 2019, S. 15-16 und 22, 7.2.2019, http://dx.doi.org/10.2139/ ssrn.3333238 (19.12.2019); B. Lobo, M. R. Alam, B. E. Whitacre: Broadband speed and employment rates, in: Telecommunications Policy 43. Jg. (2019), im Erscheinen, S. 2; D. Quaglione, M. Agovino, C. Di Berardino, A. Sarra: Exploring additional determinants of fixed broadband adoption, in: Economics of Innovation and New Technology, 27. Jg. (2018) H. 4, S. 311-312; M. Svigelj, N. Hrovatin, J. Zoric, a. a. O., S. 1002-1003 und 1013; M. Svigelj, N. Hrovatin, a.a. O., S. 409; N. E. Urama, O. Ogbu: Evaluating consumer perception and willingness to pay for broadband in Nigeria, in: Telecommunications Policy, 42. Jg. (2018), H. 6, S. 430-431. 
Dies ist verteilungspolitisch problematisch. Ähnlich ist nicht zu erkennen, warum auch profitable KMU oder KMU, für die ein besonders schneller Internetzugang kein wichtiger Treiber ihres Geschäftserfolgs ist, aus Steuermitteln Zuschüsse für die Nutzung von FTTB/H-Anschlüssen erhalten sollen. Die Mehrheit der geförderten Haushalte und profitabel operierende KMU dürften finanziell so ausgestattet sein, dass sie einen FTTB/H-Vertrag problemlos ohne staatliche Hilfe finanzieren können.

Steuergelder werden damit nicht zuletzt deshalb ineffizient verwendet, weil gerade Begünstigte mit hohem Einkommen bzw. hoher Profitabilität auch ohne Förderung einen Gigabit-Anschluss nachgefragt hätten. So gaben in Großbritannien immerhin 29 \% von 574 KMU, die im Rahmen des "Connection Voucher Scheme“ 2014 oder 2015 einen Gutschein eingelöst hatten, in einer schriftlichen Befragung an, dass sie auch ohne diese Subvention ihre Anschlussgeschwindigkeit erhöht hätten. ${ }^{18}$ Das von Gutschein-Befürwortern vorgetragene Argument, dass solche "Mitnahmeeffekte“19 bei Voucher-Programmen dadurch vermieden werden könnten, dass Endnutzer einen großen Teil der FTTB/H-Anschluss- oder Vertragskosten immer noch selbst tragen müssten, überzeugt nicht. Zwar haben Gutscheine wenig Einfluss auf das Nachfrageverhalten, wenn die Höhe der nutzerseitigen Subvention von Gigabit-Anschlüssen relativ zu deren Gesamtkosten niedrig ausfällt. Bei einer positiven Entscheidung ist aber nicht nachvollziehbar, warum Endnutzer die finanziellen Vorteile eines Vouchers für einen FTTB/H-Anschluss, den sie ohnehin nachgefragt hätten, nicht „mitnehmen“ sollten.

Die beschriebenen, dem Leistungsfähigkeitsprinzip zuwider laufenden Umverteilungs- und Mitnahmeeffekte könnten durch die Begrenzung des Kreises der Subventionsberechtigten anhand von Kriterien wie Einkommens-/ Vermögenshöhe bei Privathaushalten oder Profitabilität/ Branche bei KMU verringert werden. Eine solche Programmfokussierung wird aber von Voucher-Vorkämpfern erst gar nicht erwogen. Außerdem erhöht eine starke Zielgruppeneinengung, infolge von erhöhtem Aufwand für die Prüfung der Erfüllung von Fördervoraussetzungen, die Verwaltungskosten deutlich. Gutschein-Programme stecken somit in dem Dilemma, dass höhere Verteilungsgerechtigkeit stets durch höhere Administrationskosten erkauft werden muss.

18 Vgl. Department for Culture, Media and Sport: Broadband connection voucher scheme impact and benefits study, London 2017, S. 10. Da viele Unternehmen die vom Auftraggeber der Befragung implizit erwünschte Antwort gegeben haben dürften, dass sie ohne Voucher eine Anschlussverbesserung nicht vorgenommen hätten, dürfte das wahre „deadweight" noch deutlich größer sein.

19 Monopolkommission: Telekommunikation 2017, a.a.O., S. 81; dies.: Staatliches Augenmaß ..., a.a. O., S. 64.

\section{Zweifelhafte externe Effekte}

Die Vorteilhaftigkeit von Gigabit-Vouchern wird damit begründet, dass Endnutzer bei ihren Überlegungen hinsichtlich des Bezugs von FTTB/H-Anschlüssen außer Acht lassen würden, dass sie mit einer positiven Entscheidung die Chancen für den Ausbau eines gesamten Ortsteils mit Glaserfaser und damit für die digitale Teilhabe aller dort ansässigen Bürger/Unternehmen sowie die volkswirtschaftlichen Perspektiven Deutschlands im internationalen Standortwettbewerb verbessern. ${ }^{20}$ Deshalb sei es gesamtgesellschaftlich sinnvoll, Konsumenten und KMU durch Gutscheine dazu anzureizen, FTTB/H-Anschlüsse nachzufragen. Inwiefern die unterstellten positiven externen Effekte tatsächlich eintreten, ist allerdings höchst ungewiss. So spricht die vorgesehene niedrige absolute Höhe der Voucher pro direkt Begünstigtem dafür, dass deren Verfügbarkeit allenfalls einen sehr geringen Einfluss auf FTTB/H-Ausbauentscheidungen von Netzbetreibern haben wird. Weiter gibt es für die speziell FTTB/H-Anschlüssen zugeschriebenen positiven gesamtwirtschaftlichen Wirkungen (BIP-, Beschäftigungs-, Einkommensanstieg) keine wissenschaftlich tragfähigen empirischen Belege. ${ }^{21}$ Die Kausalkette von der FTTB/H-Anschlussverfügbarkeit über deren Take-Up-Rate bis hin zur volkswirtschaftlichen Wettbewerbsfähigkeit und der Lebenszufriedenheit der Bewohner Deutschlands ist lang. Sie wird von zahlreichen weiteren Faktoren (z. B. Konjunktur, Fachkräfteverfügbarkeit, individuelle Gesundheit) beeinflusst. Der bloße Verweis auf potenzielle Wohlfahrtsgewinne durch FTTB/H-Netze reicht zur schlüssigen Rechtfertigung von Voucher-Programmen nicht aus.

Außerdem verschweigt die Argumentationsfolie, dass Gutschein-Programme mit zahlreichen Wünschen anderer Interessengruppen nach staatlicher Unterstützung gesellschaftlich (vermeintlich) wertvoller privater oder gewerblicher Investitionen, wie etwa batterieelektrisch angetriebene Pkw oder Windkraftanlagen, konkurrieren. Um die Forderung nach Gigabit-Vouchern stichhaltig(er) vorzutragen, ist es unumgänglich, zu erörtern, inwiefern die Gutscheine stärkere positive Wohlfahrtseffekte haben als Staatshilfen in anderen Bereichen. Eine solche Diskus-

20 Vgl. exemplarisch W. Briglauer, P. Schmitz, a.a. O., S. 7-8.

21 Diese Forschungslücke resultiert auch daraus, dass Angebot und Vermarktung von FTTB/H-Netzen noch über keine lange Historie verfügen. Vgl. zur Absicherung von externen Wohlfahrtseffekten von FTTB/H-Anschlüssen durch empirische Studien zusammenfassend I. Bertschek et al.: The economic impacts of broadband Internet, in: Review of Network Economics, 14. Jg. (2015), H. 4, S. 222-224; W. Briglauer, K. Gugler: Go for gigabit?, Mannheim 2018, S. 6 und 26; I. Godlovitch et al.: The benefits of ultrafast broadband deployment, Bad Honnef 2018, S. 58-67 und 73; J. Li, M. Forzati: The social-economic impact of fiber broadband, Kista 2018, S. 1, https://ieeexplore. ieee.org/stamp/stamp.jsp?arnumber=8473582 (19.12.2019); B. Lobo, M. R. Alam, B. E. Whitacre, a.a. O., S. 4. 
sion führen die Unterstützer von Voucher-Programmen aber bisher nicht.

\section{Unnötige Bürokratiekosten}

Die durch Gigabit-Gutscheine angestrebte verbesserte Wirtschaftlichkeit des FTTB/H-Ausbaus lässt sich bei deutlich niedrigeren Kosten für die Verwaltung des Subventionsprogramms durch Integration von Nachfrageaspekten in etablierte angebotsseitige Fördermaßnahmen erreichen. Um Anschluss-Voucher einzubeziehen, sind bei der Kalkulation einer Wirtschaftlichkeitslücke bzw. des notwendigen Subventionsbedarfs einfach die Restkosten der Stichstrecken zu addieren, die vom Netzerbauer unter Berücksichtigung des Nachfragewachstums durch niedrigere Preise für den Hausanschluss zu tragen sind, nachdem er Gebäudeeigentümern den Teil der Ausbaukosten für diese Strecke, die nach der Voucher-äquivalenten Anschlusspreisabsenkung übrig bleiben, in Rechnung gestellt hat. Um Wirtschaftlichkeitseffekte von VertragsVouchern zu berücksichtigen, sind bei der Bestimmung der Subventionshöhe für ein Ausbaugebiet die Erlöse anzusetzen, die sich aus den prognostizierten höheren TakeUp-Raten ergeben, wenn man die Endkundenvertragspreise äquivalent zu den Vouchern reduzieren würde.

Durch dieses Vorgehen fließen ausbauenden Kommunen und (von innen beauftragten) Netzbetreibern im Rahmen von angebotsseitigen Subventionsprogrammen des Bundes und der Länder für Breitbandanschlüsse in unterversorgten Gebieten direkt ohne den Umweg über Gebäudeeigentümer oder Endnutzer zusätzliche Finanzmittel zu, die möglichen Einnahmen aus Anschluss- und Vertrags-Vouchern etwa entsprechen. Die Mittelzuteilung und die Überwachung der Mittelverwendung können über die heute bereits eingesetzte angebotsbezogene Förderbürokratie (z. B. beim Bundesförderprogramm Breitband: atene KOM) vorgenommen werden. Hingegen ist bei Vouchern mit viel höheren Bürokratiekosten zu rechnen, weil die Verwaltung der Subventionen durch zuvor nicht in die Breitband-Förderung maßgeblich involvierte staatliche Stellen, wie das BAFA oder die KfW, die hierfür über keine eingespielten Prozesse verfügen, erfolgen soll.

\section{Perspektiven}

Alles in allem spricht viel dafür, dass die Schwächen von Gigabit-Vouchern zur Förderung von FTTB/H-Anschlüssen in Deutschland deren Vorteile mehr als aufwiegen. Bundes- und Landesregierungen sind daher gut beraten, auch künftig keine signifikanten Mittel für Gutschein-Programme bereitzustellen.

Anstatt nach weiteren Subventionen zu rufen, haben es Netzbetreiber selbst in der Hand, Take-Up-Raten von Glasfaseranschlüssen dadurch zu steigern, dass sie von allzu ambitionierten Profitabilitätszielen Abstand nehmen und die hohen monatlichen Nutzungsentgelte für Gigabit-Anschlüsse senken, die sich im Privatkunden-Markt derzeit meist zwischen 90 Euro und 120 Euro bewegen. Der Verzicht auf „PremiumPreise" muss keineswegs zwingend zur Unwirtschaftlichkeit von FTTB/H-Investitionen führen, wenn Veränderungen staatlicher Bauvorschriften im Verbund mit der Digitalisierung von Baugenehmigungsprozessen dazu beitragen, Netzausbaukosten zu senken. Darüber hinaus können die Wirtschaft generell, die Medienbranche im Besonderen und die öffentliche Hand durch Informations-/Beratungskampagnen sowie attraktive Anwendungen, z. B. in Bereichen wie Gesundheits-, 8K-Video-Streaming- oder eGovernment-Diensten, Nutzeneinschätzungen von $\mathrm{FTTB} / \mathrm{H}$-Anschlüssen bei privaten Haushalten und KMU so beeinflussen, dass sie sich für entsprechende Angebote stärker als in der Vergangenheit begeistern. ${ }^{22}$ Schließlich sollte die Bundesregierung durch mehr Realitätssinn bei der Kommunikation von Plänen für einen Vollausbau Deutschlands mit Gigabit-Anschlüssen in zeitlicher Hinsicht überzogenen Versorgungserwartungen der Bürger und der Wirtschaft entgegenwirken. Bevor ein Marktversagen, das Gigabit-Voucher vertretbar macht, unterstellt wird, liegen - entgegen dem in Deutschland zu beobachtenden Trend zur Ausweitung von staatlichen industrie- und sozialpolitischen „Fürsorgemaßnahmen“ - zur nachfrageseitigen Förderung von FTTB/H-Anschlüssen Initiativen näher, die von einem größeren Vertrauen in die positiven Wohlfahrtseffekte sich weitgehend frei entfaltender Marktkräfte geprägt sind.

22 Vgl. übereinstimmend I. Henseler-Unger: Ausbau im Spannungsfeld von privatwirtschaftlicher Initiative und politischen Zielen, in: ifo Schnelldienst, 71. Jg. (2018), H. 7, S. 18.

Title: Vouchers For Fiber Access Lines: Why German Federal and State Governments Should Abstain From Introducing A New Subsidy Program

Abstract: Gigabit vouchers are a state-paid subsidy, which recompenses households and small and medium enterprises for a portion of the costs they incur through subscribing to Fiber-To-The-Building/-Home (FTTB/H) access offers. Various sources claim that such vouchers are an attractive means to increase demand for and supply of FTTB/H lines in Germany. However, the subsidy is plagued by serious flaws: vouchers are likely to attract only a marginal number of additional customers; to have undesired distributive side effects; to trigger minimal welfare gains; and to generate unnecessary administration costs. Thus, German federal and state governments are well advised to refrain from introducing gigabit vouchers.

JEL Classification: D62, H20, L96 